\title{
fluidización de las partículas sólidas y sus aplicaciones
}

\author{
(La fluidisation des particules solides et ses applications) \\ P. RE B O U X \\ De cL'usine nouvelle», año $12, n^{\circ} 5 \mathrm{r}$, diciembre 1956 , págs. $3 \mathrm{I}$.
}

La fluidización es el proceso mediante el cual pequet̃as partículas sólidas se mantienen en suspensión por efecto de una corriente fluída ascendente. Este fluído xportador» puede ser un gas o un líquido, siendo el arrastre por gas el de uso más frecuente $y$, en lo sucesivo, nos limitaremos prácticamente a este caso. La capa así obtenida es el lugar de movimientos, a la vez rápidos y desordenados, que recuerdan groseramente la agitación de las burbujas de vapor en el seno de una masa liquida en ebullictón; de este aspecto curioso ha resultado la terminología del fenómeno. 
Esta agitación desordenata lieva consigo un contacto intimo entre las dos fases, gaseosa y sólida, que constituyen la capa tluidizada: en el mismo orden de ideas, la superficie de contacto entre el gas y las partículas es considerabie. El interés del procedimiento reside en dos ceracterísticas esenciales:

- la intensidad de cambios térmicos o físjco-quimicos entre el gas $y$ las partículas;

- la homogeneidad de los tamaños, en el interior de la capa, caracterizan la operación industrial (temperatura, composición química del gas y del sólido, etc...); por otra parte, esta propiedad es consecuencia de la precedente.

De estas dos características primordiales se deduce el carácter pseudo-líquido

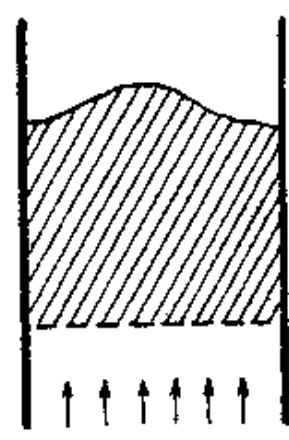

(a) CORRIENTE

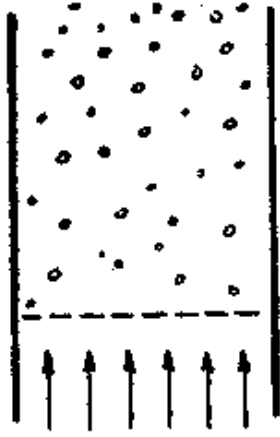

(b) VORRIENTE

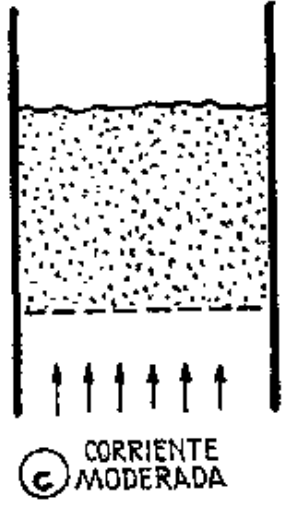

Fig. 1. del conjunto, que simplifica considerablementc el traslado de los productos sólidos. Estas capas se pueden manejar como si fueran líquidas.

\section{DESCRIPCIÓN GENERAL. LIMTTES}

El esquema de la figura i tiene la ventaja de situar la fluidización entre dos fenómenos más conocidos; asi, pues, consideraremos, para simplificar, un material constituído por esferas iguales reposando sobre una placa porosa o una rejilla de malla muy fina, a través de la cual circula una corriente de fluido ascendente (por ejemplo aire).

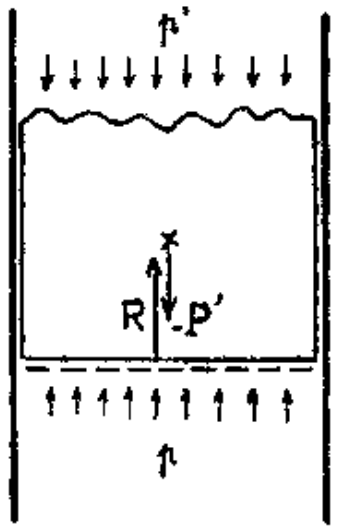

Fig. 2.

I. Para débiles velocidades del gas, la masa sólida conserva su aspecto comfacto y cada una de las partículas queda fija (croquis a). A través de la capa el aire sufre una cierta caida de presión $\Delta p$, y ello es debido:

- a los frotamientos sobre las paredes del recipicnte (esta causa es despreciable con respecto a las dos siguientes, debido a la pequeña superficie de contacto; en to que sigue, no la tendremos en cuenta);

- a los frotamientos sobre la superficie de los granos;

- a las sinuosidades que impone a cada fillete gaseoso la presencia del sólido.

No puede $\Delta p$ sobrepasar un cierto valor. Las fuerzas que actúan sobre el conjunto compacto de granos sólídos son en efecto (figura 2):

- el resultado de las fuerzas de presión, es decir, el producto $s \times \Delta p$ de la sección recta $s$ del recipiente por la diferencia $\Delta p=\not p-p^{\prime}$ de las presiones del aire a la entrada y a la salida de la capa;

- la reacción $R$ de la rejilla;

- el peso aparente $-p^{\prime}$ de la masa sólida, es decir, su peso real disminuido en el empuje de flotación.

El conjunto de estas fuerzas tiene una suma nula; como $R$, reacción de la rejitla, es necesariamente positiva resulta, que $\Delta \neq<\frac{\mathrm{P}^{\prime}}{\mathrm{s}}$.

Designaremos por «capa fija 0 alecho fijo, la disposición asi descrita, de utilidades bien conocidas. Se emplea notablemente en los hogares de rejilla, en instalaciones de tratamiento cátalítico, etc.. . 
2. Para muy grandes velocidades gaseosas, los granos son, por el contrario, arrastrados en el sentido de la corriente saliendo del recipiente, obteniéndose un estado muy disperso en el que el sólido no ocupa más que del i al $2 \%$ del volumen total (figura $I$, croquis b).

Esto ocurre cuando la velocidad $v$ del fluído sobrepasa la avelocidad límite $v_{1}$ de cada grano, es decir, la que alcanza en caida libre en el medio gaseoso considerado, supuesto en repaso. La dimensión de $v_{1}$ depende evidentemente de las variables que caracterizan al sólido y al fluido (densidades, viscosidad, diámetro de las particulas). Viene dada por fórmulas empíricas, siendo la de Stokes la más conocida, referida a partículas esféricas de muy pequeño diámetro.

Cada partícula se encuentra, de este modo, animada de una velocidad ascendente $v-v_{1}$; esto constituye el «transporte neunáticos. Ha recibido numetosas apicaciones: quemadores de carbón pulverizado, elevadores utilizados en los sìlos de cereales, cementerías, etc...

3. Entre estos limites extremos, la experiencia demuestra que existen velocidades gaseosas capaces de comunicar a los granos sólidos una cierta energía cinética, sin que, por ello, les lleve fuera del recipiente. Sin quedarse en reposo camo en el primer caso, las particuias no tienen desplazamiento de conjunto; su movimiento desordenado es comparable a la agitación molecular, a escala diferente. En este caso, la movilidad de los granos produce una dilatación de la masa sólida, puesto que, en su posición injeíal, las pequefias esferas estando juntas no son susceptibles de ningún movimiento.

Por esta analogía con la agitación molecular, la capa es muy semejante a la de un fluído. En efecto, como habiamos senalado, la mezcla sólido-gas así obtenida se comporta sensiblemente como un liquido. Se forma en su parte superior una superficie libre, más o menos perturbeda, pero cuyo plano medio es horizontal, cualquiera que sea la posición inicial del sólido. Además, por un orifício en la parte inferior, podemos tras vasar una parte de la capa, saliendo de esta forma gor acción de la gravedad, de igual modo que un liquido. Aún podemos sumergir en la mezcla un obstáculo ligero, modelo reducido de barco por ejemplo, el cual flota y toma movinicntos muy análogos a los de una nave en un mar agitacto.

E1 procedimiento que carisiste en dar a un polvo sólido las propiedades de un líquido, es a lo que llamamos flutidización. Se dice que se ha obtenido una scapa fluidizada o bien un "lecho fluidizado*. Esto aparece como un término medio entre la capa fija de granos sólidos y la capa de granos muy dispersos formada por fuerte corriente gaseosa. Debemos, pues, considerar tstos dos fenómenos extremos como limites de estudio mecánico de la Iluidización.

\section{EXPLICACIÓN BREVE}

Volvamos al experimento de la figura I, caracterizando la corriente gaseosa por su avelocidad en el vacío, $v$ : designamos de este modo al cociente del gasto $q$, por el área $s$ de la sección recta $v=\frac{q^{*}}{s}$.

Esta es la velocidad que asegurará el mismo gasto en el recipiente, en ausencia del material sólido.

En la capa fija, el volumen no ocupado por el sólido es pequeño, y lo mismo ocurre con la sección real que se ofrece al paso de los filetes gaseosos. La velocidad intersticial es, puas, en cada punto muy superior a $v$.

Si se aumenta el gasto (por consiguiente $v$ ), crece la velocidad intersticial, aumenta el gradiente en ta vecindad de cada partícula, y las fucrzas de viscosidad que él arrastra. Llega un momento donde éstas son suficientes para que los granos tengan tendencia a abandonar la capa.

Esto hace que la fracción de volumen no ocupada por el sólido aumente, lo que crece la sección de paso ofrecida al fluído, disminuyendo, por consiguiente, la velocidad intersticial. Las fuerzas de viscosidad disminuyen también, las particulas que tenderían a elevarse caen, $y$ así sucesivamente.

De este modo, en el interior del recipiente se establece un equilibrio mediante el cual cada grano está sometido a dos fuerzas de sentido contrario: su peso y la resultante ascendente de las fuerzas de viscosidad. Se obtiene de tal suerte un conjunto de partículas separadas que constituye precisamente el lecho fluidizado. El fenómeno resulta muy complicado por los choques entre las partículas mismas y la pared.

Despreciando las fuerzas tangençiales debidas al frotamiento con la paled del recipiente, es fácil demos- 
trar que la caida de presión sufrida por el fluido a través de la capa fluidizada es igual al cociente del peso aparente $\mathrm{P}$ de la masa sólida por el área $s$ de la sección recta.

Podemos en efecto reanudar el razonamiento desarrollado a propósito de la capa fija, suponiendo nula la reacción $R$ de la rejilla, puesto que, teóricamente, las partículas no están muy unidas y no reposan sobre la rejilla.

La mediana del vector velocidad de cada grano siendo nula, el centro de gravedad del sistema queda fijo. La relación fundamental de la estática (suma de las fuerzas igual a cero) es aún valedera, aunque el sólido esté en movimiento; ella da: $\Delta p=\frac{\mathrm{P}^{\prime}}{s}$

A través de la masa fluidizada, la caida de presión es pues independiente de la velocidad, e jgual al peso de la capa por unidad de área de la sección recta. Un aumento de la velocidad del gas se traduce-solamente en una dilatación suplementaria.

\section{DIVERSOS ASPECTOS Y TERMINOLOGIA}

Contrariamente a lo que pudieran dejar inducir las consideraciones precedentes, por demasiado esquemáticas, la capa constituida por particulas aisladas y regularmente distribuídas no es el caso general; puede decirse que no se da jamás en tluidización gaseosa. Según la velocidad del fluido, la naturaleza del sólido.y sobre todo la complejidad de la forma de los granos, se obtienen estados de fluidización muy variados, que se muestran más o menos interesantes desde el punto de vista industriaj, según la operación a realizar. No es pues inútil, atunque parezca ingenuo, mencionar que el estudio de estos diversos estados ha sido retardado considerablemente por cuestiones de terminología. Los técnicos franceses han sido en principio llevados a error por términos utilizados en publicaciones americanas, y tan sólo a pacientes esfuerzos y observaciones experimentales muy minuciosas es por lo que han podido librarse del vocabulario empleado por los anglosajones.

La hetereogeneidad de un lecho fluidizado es pues regla general; la fase menos acusada es la llamada de "burbujeon: burbujas gaseosas de pequefas dimensiones que nacen, numerosas y muy juntas, en la base del aparato. Ellas suben lentamente, agrandándose a medida que ascienden, rompiéndose, finalmente, al llegar a la superficie, deformándola y conservándose, sin embargo, en un plano medio horizontal y fijo, en el curso del tiempo. Este aspecto de la fluidización es en general lo que se investiga en las operaciones industriales.

Puede también ocurrir que el gas encuentre en la masa sólida trayectorias de menor resistencia u oquedades a través de las cuales se infiltra, disminuyendo el gasto en el resto de la capa. Estas trayectorias son, por otra parte, muy inestables y se desplazan continuamente, pero los registros cinematográficos a cámara lenta han permitido mostrarlas claramente (CERCHAR, laboratorio de Verneuil).

Cuando la longitud de ciertas burbujas alcarzan el diámetro del recipiente, se obtiene el cefecto de èmbolos: el agregado sólido comprendido entre dos huecos consecutivos se eleva lentamente en un solo bloque, de igual forma que un pistón lo hace en un cilindro.

Después de un cierto trayecto, la burbuja inferior encuentra un hueco y como el pistón, ésta se precipita bruscamente.

Este fenómeno no se produce en los reactores de gran diámetro. A fuertes velocidades, la agitación, no obstante, toma una amplitud tal que se observa, en la superficie de separación, verdaderos geysers: los paquetes de partículas sólidas son violentamente proyectados hacia arriba para volver a caer enseguida en forma de paraguas. Llega a ser muy difícil definir un plano medio de interfase; esto es el aburbujeo tumultuoso».

El límite entre estos diversos estados no está claro, y todos tos intermedios son posibles.

Los factores que determinan el estado de la fluidización son muy numerosos. El más importante es la velocidad de la corriente gaseosa: en un aparato experimental de $190 \mathrm{~mm}$ de diámetro, en el instante de la fluidización de una capa de carbón de $330 \mathrm{~mm}$ de alzura, hemos obterido los siguientes resultados:

- para $v=245 \mathrm{~cm} / \mathrm{s}$, la fiuidización comienza;

- para $v$ comprendida entre 26 y $29 \mathrm{~cm} / \mathrm{s}$, el burbujeo regular; 
- para $v$ comprendida entre 29 y $32 \mathrm{~cm} / \mathrm{s}$, estados intermedios, $y$

- para $v=32 \mathrm{~cm} / \mathrm{s}$, comienza el sefecto de émbolo».

E1 estado de la capa depende, de igual modo, de las dimensiones del recipiente y de otros numerosos factores. De una forma general, un lecho fluido se caracteriza por una constante inestabilidad; en condiciones operatorias idénticas, se llegan a obsevar estados muy diferentes. Todo esto no está hecho para acelerar el desarrollo del procedimiento: la utilización es siempre muy delicada cuando se trata de construir un equipo de gran dimensión si no se tiene la certeza previa del resultado.

\section{APLICACIONES}

Desde 1945, la fluidización ha tomado una extensión considerable en todas las ramas de la actividad; antes de enumerarlas, vamos a dar algunas precisiones sobre la forma en que deben ser aplicados estos procedimientos en los casos más sencillos.

El caso más usado es el de la fluidización discontinua. El proceso es muy análogo al que hemos descrito

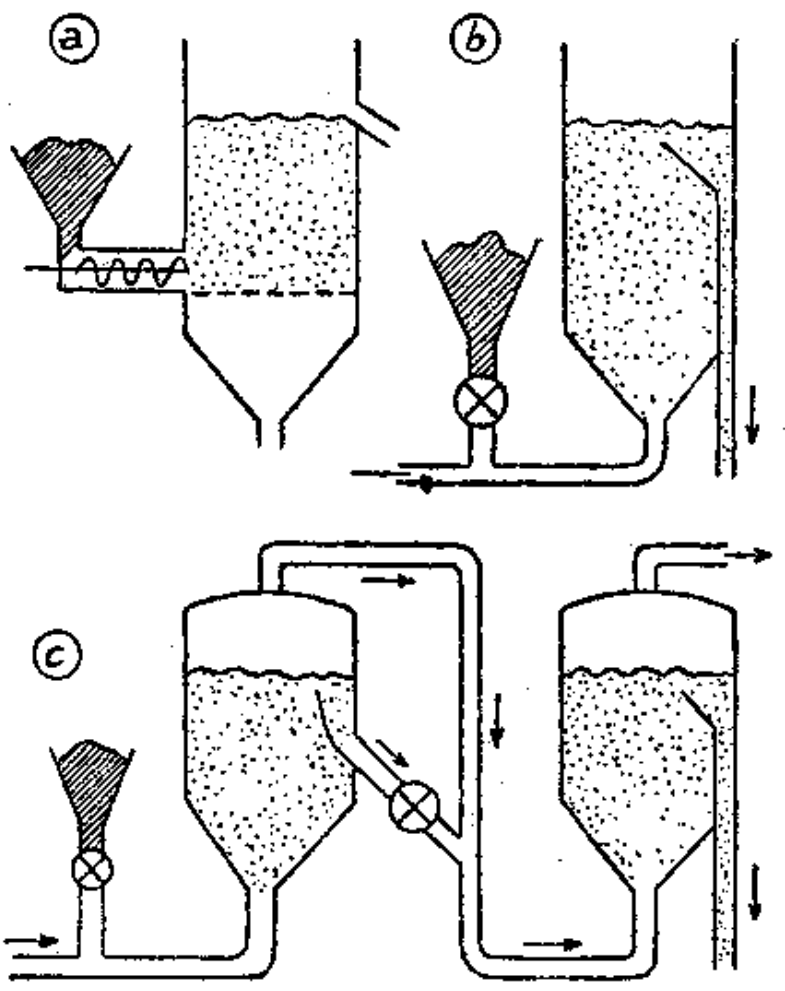

Fig. 3. al principio. La carga sólida, que reposa sobre una rejilla, se pone en suspensión por la corriente de gas que entra. Cuando la reacción ha tcrminado, se para la corriente gaseosa, el aparato se vacia y luego se llená con carga reciente.

Es lamentable no aprovecharse de la fluidez de las capas para facilitar el aprovisionamiento y poder realizar una operación continua; el producto tratado es retirado del reactor a medida que nuevas cantidades de carga recientes son introducidas. Sobre la fig. 3 el esquema representa tina alimentación clásica en espirai, el sólido tratado sale por la parte superior. El croquis $b$ representa la soltición adoptada en la mayor parte de los casos, el material cae por acción de la gravedad desde una tolva $\mathrm{T}$ hasta la conducción, donde se introduce el fluibo, y el conjunto fluído sólido sale por esta canalización (transporte neumático) hasta el recipiente de tratamiento; esta disposición suprime la rejilla. Los graros tratados salen por un orificio adecuado, a cuya salida pueden ser trasiadados por otra corriente gaseosa o cargados en vagones, etc.

Un obstáculo aparece inmediatamente: por razón de la gran agitación de las partículas, una cualquiera entre ellas tiene tantas posibilidades de salir como las demás, aunque se encuentre en la capa desde muy poco tiempo. La duración media $t_{0}$ de permanencia de las partículas es evidentemente igual al cociente entre la masa de la capa y el gasto, pero la permanencia real de cada una puede variar de cero a infinito. El cálculo muestra que la probabilidad $p$ para que un grano quede en el lecho durante un tiempo superior a $t$ viene dado por: $p=e^{-t / t_{0}}$.

Para mitigar este inconveniente, se ha recurrido algunas veces a la fluidización sescalonada", es decir, cuando la operación industrial se realiza en varios estados. En el caso de dos reactores en serie (croquis c) con una duración media $t_{0} / 2$ idéntiça en cada recipiente, el cálculo da:

$$
p=\left(1+\frac{t}{t_{0}}\right) e^{-\frac{t}{s_{0}}}
$$


Ĺa tabia que exponemos a continuación da los valores de $t$, en los dos casos, para una misma permanencia media de 25 minutos

\begin{tabular}{c|c|c}
\hline$t$ (minutos) & $\rho$ (1 reactor) & (9 reactores) \\
\hline & & \\
\hline 0,25 & 0,991 & 0,9998 \\
2,5 & 0,905 & 0,9825 \\
5 & 0,82 & 0,770 \\
12,5 & 0,61 & 0,736 \\
25 & 0,37 & 0,408 \\
125 & 0,007 & 0,0005 \\
\hline
\end{tabular}

Así, en el primer caso, $0,9 \%$ de Jas particulas quedarán sometidas al tratamitnto menos de un cuarto de minuto; en el $2 .^{\circ}$ caso, esta proporción decae al $0,02 \%$.

La fluidización escalonada posee otra ventaja: permite prever una circulación de conjunto del sólido de sentido contrario a la del gas, lo que asegura entre los dos cuerpos cambios de calor más racionales.

Los reactores pueden ser distintos como indica ia figura, pero, generalmente, constituyen un conjunto único, las diversas capas son soportadas por rejillas superpuestas, a través de las cuales pasa sucesivamente la corriente gaseosa. El sófido cae por gravedad de una capa a la otra, gracias a los tubos de rebosadero.

Entre las aplicaciones de la fluidización, citaremos el tratamiento químico de los combustibles sólidos (principalmente el cock pulverulento, los carbones activos, $y$ en el dominio de la combustión, el hogar para la quema de fluídos «Ignifluids), el tratamiento de los combustibles gaseosos, de los hidrocarburos líquidos, de los minerales metálicos, transporte de sólidos, secado y la clasificación de granos, etc.

(Tomado de ta *Revista de industria mineraln Junio 1956, P. Reboux, subdirector de la Escuela Nacional Superior de Minas de Saint-Etienne). 